\title{
Heterogeneity of asthma and COPD overlap
}

This article was published in the following Dove Press journal: International Journal of COPD

\author{
Min-Hye Kim' \\ Chin Kook Rhee ${ }^{2}$ \\ Kyungjoo $\mathrm{Kim}^{2}$ \\ Sang Hyun $\mathrm{Kim}^{3}$ \\ Jung Yeon Lee ${ }^{4}$ \\ Yee Hyung Kim ${ }^{5}$ \\ Kwang $\mathrm{Ha} \mathrm{Yoo}{ }^{6}$ \\ Young-Joo Cho' \\ Ki-Suck Jung ${ }^{7}$ \\ Jin Hwa Lee'
}

'Department of Internal Medicine, College of Medicine, Ewha Womans University, ${ }^{2}$ Department of Internal Medicine, Seoul St Mary's Hospital, The Catholic University of Korea, Seoul, ${ }^{3}$ Big Data Division, Health Insurance Review and Assessment Service, Wonju, ${ }^{4}$ Division of Pulmonary and Critical Care Medicine, Department of Internal Medicine, Konkuk University Chungju Hospital, Chungju, ${ }^{5}$ Department of Pulmonary and Critical Care Medicine, Kyung Hee University Hospital at Gangdong, Kyung Hee University School of Medicine, ${ }^{6}$ Department of Internal Medicine, Konkuk University College of Medicine, Seoul, ${ }^{7}$ Division of Pulmonary, Allergy and Critical Care Medicine, Department of Internal Medicine, Hallym University Medical Center, Hallym University College of Medicine, Anyang, Republic of Korea

Correspondence: Jin Hwa Lee Department of Internal Medicine, College of Medicine, Ewha Womans University, I07I, Anyangcheon-ro, Yangcheon-gu, Seoul 07985, Republic of Korea

Tel +82 226506007

Fax +82 226502076

Email jinhwalee@ewha.ac.kr
Background: Asthma and COPD are heterogeneous diseases. Patients with both disease features (asthma-COPD overlap [ACO]) are common. However, clinical characteristics and socio-economic burden of ACO are still controversial. The aim of this study was to identify the heterogeneity of ACO and to find out the subtypes with clinical impact among ACO subtypes.

Methods: In the Korean National Health and Nutrition Examination Survey (KNHANES) conducted between 2007 and 2012, subjects who were $\geq 40$ years and had prebronchodilator $\mathrm{FEV}_{1} /$ $\mathrm{FVC}<0.7$ and $\mathrm{FEV}_{1} \geq 50 \%$ predicted were included. The presence or absence of self-reported wheezing was indicated by $\mathrm{W}+$ or $\mathrm{W}-$ and used as an index of airway hyper-responsiveness. $\mathrm{S}+/ \mathrm{S}-$ was defined as subjects who were smokers/never smokers. The subjects were divided into the following four groups: $\mathrm{W}-\mathrm{S}-, \mathrm{W}-\mathrm{S}+, \mathrm{W}+\mathrm{S}-$, and $\mathrm{W}+\mathrm{S}+\mathrm{W}+\mathrm{S}-$ and $\mathrm{W}+\mathrm{S}+$ were asthma-predominant ACO and COPD-predominant ACO, respectively. KNHANES and linked National Health Insurance data were analyzed.

Results: The asthma-predominant ACO group showed the lowest socioeconomic status, FEV FVC\% predicted, and quality of life (QoL) levels. The COPD-predominant ACO group showed the highest hospitalization rate, outpatient medical cost, and total and outpatient health care utilization. COPD-predominant ACO was associated with exacerbations compared to the $\mathrm{W}-\mathrm{S}-$ group (adjusted odds ratio [aOR], 1.79; 95\% confidence interval [CI], 1.12-2.85; $P=0.015$ ) and $\mathrm{W}-\mathrm{S}+$ group (OR 2.11; 95\% CI 1.43-3.10; $P<0.001)$. COPD-predominant ACO was associated with increased medical cost.

Conclusion: Asthma-predominant ACO individuals displayed poorer socioeconomic status and QoL compared to the COPD-predominant ACO group. The COPD-predominant ACO group displayed more frequent exacerbations and greater medical costs. Considering the heterogeneity of ACO, it is desirable to identify subtypes of ACO patients and appropriately allocate limited medical resources.

Keywords: asthma, COPD, overlap, heterogeneity, health care utilization, exacerbation

\section{Introduction}

Asthma-COPD overlap (ACO) is a disorder in which features of asthma and COPD are mixed. The definition, clinical features, and course of ACO are still controversial. ${ }^{1,2}$ Although the Global Initiative for Asthma (GINA) and Global Initiative for Chronic Obstructive Lung Disease (GOLD) outlined 11 items to allow ACO diagnosis, there is no consensus on diagnostic criteria of ACO. ${ }^{3-6}$ ACO may be simply defined as COPD with airway hyper-responsiveness or as asthma with persistent airflow limitation. ${ }^{7}$

The prevalence of ACO in those with COPD and asthma is $12 \%-55 \%$, 5,7 and $16 \%-61 \%,{ }^{5,8}$ respectively. In some studies, ACO patients displayed more severe symptoms, poor quality of life (QoL), and frequent exacerbations compared to COPD patients. ${ }^{9-11}$ Contrarily, a study reported no difference in severity or mortality between ACO and COPD alone, ${ }^{7}$ with another study describing better survival in ACO than in COPD. ${ }^{12}$ Surveys in the USA, China, and South Korea reported higher absence rates, submit your manuscript Dovepress http://dx,doi,org/| 0.2
International Journal of COPD 20|8:|3 |25|-|260

(c) (i) (5) $2018 \mathrm{Kim}$ et al. This work is published and licensed by Dove Medical Press Limited. The full terms of this license are available at https.//www.dovepress.com/terms.ph C. hereby accept the Terms. Non-commercial uses of the work are permitted without any further permission from Dove Medical Press Limited, provided the work is properly attributed. For permission for commercial use of this work, please see paragraphs 4.2 and 5 of our Terms (https://www.dovepress.com/terms.php). 
medical resource utilizations, and medical costs for $\mathrm{ACO}$ patients compared to COPD patients. ${ }^{8,13,14}$ A recent prospective population-based analysis reported the heterogeneity of ACO concerning the onset of asthma. ${ }^{11}$ The reason for the differing prevalence and clinical outcomes of ACO in different studies could reflect the different definitions of ACO as well as heterogeneity in ACO. However, in order to overcome the disease, it is necessary to confirm that ACO is a disease with various characteristics. It is important to recognize that there are also various subtypes within ACO and that they may show different patterns in their frequency of exacerbations, medical use, and cost.

Therefore, the aims of this study were to investigate the heterogeneity of ACO in subjects with mild-to-moderate airflow limitation and to identify the diverse epidemiological characteristics and subtypes of ACO associated with exacerbations and health care use and cost through the national survey and insurance claims data.

\section{Methods}

\section{Study design}

In the Korean National Health and Nutrition Examination Survey (KNHANES) ${ }^{15}$ conducted between 2007 and 2012, subjects aged $\geq 40$ years with forced expiratory volume in 1 second $\left(\mathrm{FEV}_{1}\right) /$ forced vital capacity $(\mathrm{FVC})<0.7$ and $\mathrm{FEV}_{1} \geq 50 \%$ predicted were included. Self-reported wheezing was indicated as presence $(\mathrm{W}+)$ or absence $(\mathrm{W}-)$. An S+ subject was a current smoker or an exsmoker who had smoked $\geq 100$ cigarettes. An S- subject had never smoked or had smoked $<100$ cigarettes in his/her lifetime, which is also used in other surveys. ${ }^{16,17}$ The subjects were divided into the following four groups: $\mathrm{W}-\mathrm{S}-, \mathrm{W}-\mathrm{S}+, \mathrm{W}+\mathrm{S}-$, and $\mathrm{W}+\mathrm{S}+\mathrm{W}+\mathrm{S}-$ was regarded as asthma-predominant $\mathrm{ACO}$, and $\mathrm{W}+\mathrm{S}+$ was regarded as COPD-predominant ACO. KNHANES data were analyzed according to the groups. These data were linked to the National Health Insurance (NHI) data, and the use and cost of health care services from January 2007 to December 2012 for each group were analyzed. Exacerbations were defined as deterioration in respiratory symptoms requiring systemic corticosteroids and/or antibiotics for hospitalization or emergency room (ER) visits, as in previous studies. ${ }^{13,18-21}$ Medical costs and days of health care use not related to the respiratory diseases were excluded from the analysis. Detailed methods are described in previous studies ${ }^{13,18-22}$ and are presented in Figure 1.

Among the variables, household income was divided into quartiles: upper, middle upper, middle lower, and lower. The level of education was based on the graduation level (elementary school, middle school, high school, and college). Marital status was divided into single, married, divorced, and bereaved. Physical activity was divided into three levels by the questionnaire. Physical activity 1 was defined as highly

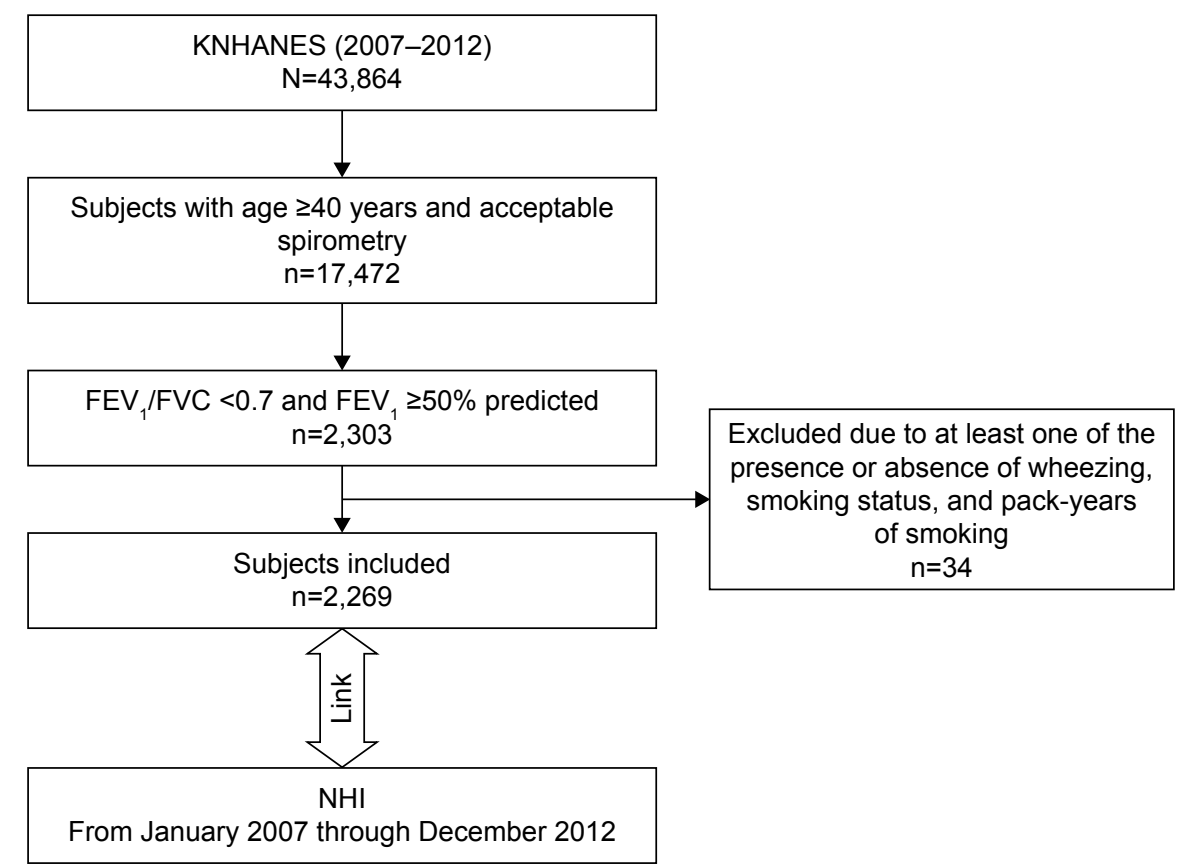

Figure I Study flow diagram.

Abbreviations: $\mathrm{FEV}_{1}$, forced expiratory volume in I second; FVC, forced vital capacity; KNHANES, Korea National Health and Nutrition Examination Survey; NHI, National Health Insurance. 
intensive activity that increases respiration or heart rate to a high level, physical activity 2 is moderate activity that leads to a slight increase in respiration or heart rate, and physical activity 3 is low-intensity activity. Self-rated health was also scored by the questionnaire and classed as excellent, good, fair, poor, and very poor. EuroQoL five dimensions questionnaire (EQ-5D) index was used to measure health-related QoL and included mobility, self-care, usual activities, pain/ discomfort, and anxiety/depression. ${ }^{23}$ Each item of EQ-5D was graded in three levels; the higher the grade, the lower the QoL. Our study was approved by the National Evidence Based Coordinating Agency Ethics Committee. Due to the retrospective nature of this study, the Ethics Committee waived the requirement for informed consent from subjects. All data accessed were de-identified.

\section{Statistical analyses}

All data are expressed as mean \pm SD or n (\%). Analysis of variance or Kruskal-Wallis test was used to compare continuous variables among the four groups, and Student's $t$-test or Mann-Whitney test was used to compare asthmapredominant and COPD-predominant ACO. Categorical variables were analyzed by chi-square test or Fisher's exact test. Multiple logistic regression analysis was used to determine the effect of groups on the deterioration that required hospitalization or emergency room visits, and multiple linear regression analysis was used to determine the effect of groups on medical costs. A four-step model was applied to adjust confounding factors. No factor was adjusted in step 1. Age, sex, and body mass index (BMI) were adjusted in step 2. Age, sex, BMI, and household income were adjusted in step 3. Age, sex, BMI, household income, and $\mathrm{FEV}_{1}$ were adjusted in step 4. Odds ratio (OR) and 95\% confidence interval (CI) or beta coefficients are presented. $P$-value $<0.05$ was considered statistically significant. All statistical analyses were performed using SAS Version 9.2 (SAS Institute Inc., Cary, NC, USA).

\section{Results}

Of the 2,269 subjects with $\mathrm{FEV}_{1} / \mathrm{FVC}<0.7$ and $\mathrm{FEV}_{1}>50 \%$ predicted, 410 (18\%) subjects with $\mathrm{W}+$ belonged to ACO. Among these 410 ACO subjects, 154 never smokers were classified as $\mathrm{W}+/ \mathrm{S}-$ (asthma-predominant $\mathrm{ACO}$ ) and 256 smokers were classified as $\mathrm{W}+\mathrm{S}+(\mathrm{COPD}$-predominant ACO). Of those without wheezing (W-), 681 were never smokers (W-/S-) and 1,178 were smokers (W-/S+). Although mean age was not different, age distribution was significantly different among the four groups $(P=0.001)$.
Subjects aged 40-50 years were more frequent in the $\mathrm{W}+$ groups and subjects aged 50-70 years were more frequent in the $\mathrm{S}+$ groups. Men were more prevalent in both $\mathrm{S}+$ groups than in both $\mathrm{S}$ - groups and in the COPD-predominant ACO group than in the asthma-predominant ACO group (both $P<0.001)$. BMI and waist circumference were the highest in the COPD-predominant ACO group (both $P<0.001$ ). Household income and education level were the highest in the $\mathrm{W}-/ \mathrm{S}-$ group among the four groups $(P<0.001)$ and were lower in the asthma-predominant ACO group than in the COPD-predominant ACO group $(P<0.001)$. There were more married subjects in the two $\mathrm{S}+$ groups and more bereaved subjects in the other $\mathrm{S}-$ groups $(P<0.001) . \mathrm{FEV}_{1}$, $\mathrm{FVC}$, and $\mathrm{FEV}_{1} / \mathrm{FVC}$ were lower in the $\mathrm{W}+$ group than in the $\mathrm{W}-$ group $(P<0.001)$. Among the $\mathrm{W}+$ groups, $\mathrm{FEV}_{1} / \mathrm{FVC}$ was lower in the COPD-predominant $\mathrm{ACO}$ group than in the asthma-predominant $\mathrm{ACO}$ group $(P=0.002)$, while $\mathrm{FEV}_{1}$ and $\mathrm{FVC}$ were not different. Based on the responses to the questionnaires, more subjects were diagnosed with $\mathrm{COPD}$ in the COPD-predominant ACO group and more subjects were diagnosed with asthma in the asthma-predominant ACO group compared to the other groups $(P<0.001)$. Based on the NHI data, osteoarthritis, osteoporosis, and depression were more common in the $\mathrm{S}-$ groups $(P=0.012,<0.001$, and $<0.001$, respectively) and osteoporosis was most prevalent in the asthma-predominant ACO group $(P<0.001)$ (Table 1).

Physical activity 1 (high intensity) was more frequent in the $\mathrm{S}+$ groups among the four groups $(P<0.001)$ and in the COPD-predominant ACO group compared to the asthma-predominant ACO group $(P=0.026)$. Regarding the self-rated health levels, both $\mathrm{W}$ - groups more often rated their health as excellent or good compared to the $\mathrm{W}+$ groups $(P<0.001)$, with health rated as poorer more often in the asthma-predominant ACO group than in the COPDpredominant ACO group $(P=0.001)$. Both $\mathrm{W}+$ groups showed poorer health-related QoL in all EQ-5D items compared to $\mathrm{W}-$ groups $(P<0.001)$, and usual activities and pain/ discomfort scores were lower in the asthma-predominant ACO group than in the COPD-predominant ACO group $(P=0.024)$ (Table 2).

NHI data revealed that insurance type was not different among the four groups. Hospitalization rate was higher in both $\mathrm{W}+$ groups $(P<0.001)$, but there was no difference between both ACO groups. Mean total medical costs did not differ appreciably among the four groups. Outpatient medical expenses were highest in the COPD-predominant ACO group $(P<0.001)$. This tendency was similar in the number of days of health care use (Table 3 ). 
Table I Epidemiological characteristics of the four groups according to the presence or absence of wheezing or smoking history

\begin{tabular}{|c|c|c|c|c|c|c|}
\hline \multirow{2}{*}{$\begin{array}{l}\text { Wheezing } \\
\text { Smoking history }\end{array}$} & \multicolumn{2}{|l|}{$\mathbf{W}_{-}$} & \multicolumn{2}{|l|}{$\mathbf{W}+$} & \multirow[t]{2}{*}{$P$-value* } & \multirow[t]{2}{*}{$P$-value** } \\
\hline & S- & $\mathbf{S}+$ & S- & $\mathbf{S}+$ & & \\
\hline Number of subjects, $n$ & 681 & $\mathrm{I}, 178$ & 154 & 256 & & \\
\hline Age (years) & $65.5 \pm 10.4$ & $64.9 \pm 9.5$ & $64.7 \pm 11.3$ & $63.5 \pm 10.5$ & 0.304 & 0.066 \\
\hline$\geq 40$ and $<50$ & $60(9)$ & $86(7)$ & $22(14)$ & $31(12)$ & 0.197 & 0.001 \\
\hline$\geq 50$ and $<60$ & $117(17)$ & $243(2 I)$ & $27(18)$ & $55(2 I)$ & & \\
\hline$\geq 60$ and $<70$ & $233(34)$ & $421(36)$ & $44(28)$ & $92(36)$ & & \\
\hline$\geq 70$ and $<80$ & $225(33)$ & $389(33)$ & $55(36)$ & $66(26)$ & & \\
\hline$\geq 80$ & $46(7)$ & $39(3)$ & $6(4)$ & $12(5)$ & & \\
\hline Sex (\% male) & $240(35)$ & $1,132(96)$ & $27(18)$ & $231(90)$ & $<0.001$ & $<0.001$ \\
\hline BMI $\left(\mathrm{kg} / \mathrm{m}^{2}\right)$ & $23.7 \pm 2.9$ & $23.3 \pm 2.7$ & $23.9 \pm 3.2$ & $24.1 \pm 2.8$ & 0.600 & $<0.001$ \\
\hline$<18.5$ & $15(2)$ & $36(3)$ & $10(7)$ & $4(2)$ & 0.057 & $<0.001$ \\
\hline$\geq 18.5$ and $<25$ & $464(68)$ & $837(7 I)$ & $92(60)$ & $16 \mid(63)$ & & \\
\hline$\geq 25$ and $<30$ & $187(28)$ & $296(25)$ & $47(30)$ & $85(33)$ & & \\
\hline$\geq 30$ & $15(2)$ & $9(0.8)$ & $5(3)$ & $6(2)$ & & \\
\hline Waist (cm) & $82.9 \pm 8.9$ & $85.0 \pm 8.0$ & $83.7 \pm 9.7$ & $86.7 \pm 8.2$ & 0.001 & $<0.001$ \\
\hline Household income & & & & & $<0.001$ & $<0.001$ \\
\hline Lower & $21(3)$ & 391 (33) & $75(49)$ & $79(3 \mathrm{I})$ & & \\
\hline Middle lower & $247(36)$ & $310(27)$ & $25(16)$ & $79(3 \mathrm{I})$ & & \\
\hline Middle upper & $162(24)$ & $240(2 I)$ & $22(14)$ & $56(22)$ & & \\
\hline Upper & $129(19)$ & $213(18)$ & $27(18)$ & $35(14)$ & & \\
\hline Education level & & & & & $<0.001$ & $<0.001$ \\
\hline Elementary school & $2(0.3)$ & $433(37)$ & $105(68)$ & $108(42)$ & & \\
\hline Middle school & 337 (49) & $219(19)$ & $16(10)$ & $49(19)$ & & \\
\hline High school & $119(17)$ & $333(28)$ & $20(13)$ & $59(23)$ & & \\
\hline College & $|4|(2 \mid)$ & $192(16)$ & $13(8)$ & $38(15)$ & & \\
\hline Marital status & & & & & $<0.001$ & $<0.001$ \\
\hline Single & $3(0.4)$ & II (0.9) & I $(0.6)$ & $4(1.6)$ & & \\
\hline Married & $496(73)$ & $1,048(90)$ & $102(66)$ & $222(87)$ & & \\
\hline Divorced & $15(2.2)$ & $44(3.8)$ & $3(1.9)$ & $7(2.7)$ & & \\
\hline Bereaved & $165(24)$ & $72(6 . I)$ & $48(3 \mathrm{I})$ & $23(9)$ & & \\
\hline \multicolumn{7}{|l|}{ Smoking history } \\
\hline Exsmoker & $20(3)$ & $549(47)$ & $5(3)$ & $149(58)$ & & \\
\hline Current smoker & $101(15)$ & $629(53)$ & $12(8)$ & $107(42)$ & & \\
\hline Never smoker & $560(82)$ & 0 & 137 (89) & 0 & & \\
\hline Smoking (pack-years) & $0.3 \pm 0.9$ & $33.3 \pm 21.8$ & $0.2 \pm 0.7$ & $32 \pm 20.3$ & $<0.001$ & $<0.001$ \\
\hline \multicolumn{7}{|l|}{ Spirometry } \\
\hline $\mathrm{FEV}, \%$ predicted & $80.0 \pm 14.4$ & $80.2 \pm 12.9$ & $72.8 \pm 13.8$ & $73.0 \pm 13.0$ & 0.855 & $<0.001$ \\
\hline FVC\% predicted & $90.9 \pm 14.2$ & $91.5 \pm 13.1$ & $86.7 \pm 13.5$ & $88.0 \pm 13.1$ & 0.335 & $<0.001$ \\
\hline $\mathrm{FEV}_{1} / \mathrm{FVC}$ & $0.65 \pm 0.05$ & $0.64 \pm 0.06$ & $0.63 \pm 0.06$ & $0.6 I \pm 0.08$ & 0.002 & $<0.001$ \\
\hline \multicolumn{7}{|c|}{ Past history of respiratory or allergic diseases } \\
\hline COPD & $5(0.7)$ & $14(1.2)$ & $4(2.6)$ & $17(6.6)$ & 0.072 & $<0.001$ \\
\hline Asthma & $34(5)$ & $34(2.9)$ & $61(39.6)$ & $62(24.2)$ & 0.001 & $<0.001$ \\
\hline COPD and asthma & $I(0.1)$ & $I(0.1)$ & $4(2.6)$ & $8(3.1)$ & 0.759 & $<0.001$ \\
\hline Tuberculosis & $81(12)$ & $144(12.3)$ & $18(11.7)$ & $42(16.4)$ & 0.191 & 0.267 \\
\hline Atopic dermatitis & $7(1)$ & $15(1.3)$ & $5(3.2)$ & $2(0.8)$ & 0.062 & 0.135 \\
\hline \multicolumn{7}{|l|}{ Comorbidities } \\
\hline Hypertension & $385(57)$ & $643(55)$ & $90(58)$ & I5I (59) & 0.914 & 0.507 \\
\hline Diabetes mellitus & $262(38)$ & $449(38)$ & $56(36)$ & $113(44)$ & 0.121 & 0.293 \\
\hline Cerebrovascular disease & $311(46)$ & $507(43)$ & $67(44)$ & II 2 (44) & 0.962 & 0.746 \\
\hline Osteoarthritis & $95(14)$ & $110(9)$ & $22(14)$ & $32(12)$ & 0.605 & 0.012 \\
\hline Osteoporosis & $264(39)$ & $158(13)$ & $74(48)$ & $44(17)$ & $<0.001$ & $<0.001$ \\
\hline Depression & $146(2 \mid)$ & $167(14)$ & $34(22)$ & $46(18)$ & 0.309 & $<0.001$ \\
\hline Hyperlipidemia & $254(37)$ & $422(36)$ & $57(37)$ & $99(39)$ & 0.738 & 0.819 \\
\hline Lung cancer & $19(3)$ & $33(3)$ & $3(2)$ & $6(2)$ & 0.791 & 0.914 \\
\hline Coronary artery disease & 161 (24) & $258(22)$ & $46(30)$ & 7I (28) & 0.705 & 0.054 \\
\hline
\end{tabular}

Notes: Data are presented as mean \pm SD or number (\%). Comorbidities based on NHI data. *Comparison between S- and S+ groups within the $\mathrm{W}+$ groups. $* *$ Comparison among the four groups.

Abbreviations: BMI, body mass index; $\mathrm{FEV}_{1}$, forced expiratory volume in I second; FVC, forced vital capacity; NHI, National Health Insurance; S, smoking; W, wheezing. 
Table 2 Physical activity and health-related quality of life of the four groups with mild-to-moderate airflow limitation

\begin{tabular}{|c|c|c|c|c|c|c|}
\hline \multirow{2}{*}{$\begin{array}{l}\text { Wheezing } \\
\text { Smoking history }\end{array}$} & \multicolumn{2}{|l|}{$\mathbf{W}-$} & \multicolumn{2}{|l|}{$\mathbf{W}+$} & \multirow[t]{2}{*}{$P$-value* } & \multirow[t]{2}{*}{$\boldsymbol{P}$-value** } \\
\hline & $\mathbf{S}-$ & $\mathbf{S}+$ & $\mathbf{S}-$ & $\mathbf{S}+$ & & \\
\hline Number of subjects, $n$ & 681 & $\mathrm{I}, \mathrm{I} 78$ & 154 & 256 & & \\
\hline Physical activity I & $136(20)$ & $319(27)$ & $32(2 I)$ & $79(31)$ & 0.026 & $<0.001$ \\
\hline Physical activity 2 & $234(34)$ & $414(35)$ & $57(37)$ & $90(35)$ & 0.704 & 0.939 \\
\hline Physical activity 3 & $558(82)$ & $990(84)$ & $122(79)$ & $220(86)$ & 0.077 & 0.213 \\
\hline \multicolumn{7}{|l|}{ Self-rated health } \\
\hline Excellent & $31(5)$ & $71(6)$ & $4(3)$ & $5(2)$ & 0.001 & $<0.001$ \\
\hline Good & $205(30)$ & $382(32)$ & $22(14)$ & $51(20)$ & & \\
\hline Fair & $255(37)$ & $465(40)$ & $42(27)$ & $108(42)$ & & \\
\hline Poor & $143(21)$ & $215(18)$ & $68(44)$ & $79(3 I)$ & & \\
\hline Very poor & $45(7)$ & $44(4)$ & $18(12)$ & $13(5)$ & & \\
\hline \multicolumn{7}{|c|}{ Health-related quality of life } \\
\hline \multicolumn{7}{|c|}{ EQ-5D level } \\
\hline \multicolumn{7}{|l|}{ Mobility } \\
\hline I & $453(67)$ & $940(80)$ & $85(55)$ & $172(67)$ & 0.052 & $<0.001$ \\
\hline 2 & $218(32)$ & $230(19)$ & $66(43)$ & $80(31)$ & & \\
\hline 3 & $8(1)$ & $7(0.6)$ & $3(2)$ & $4(1.6)$ & & \\
\hline \multicolumn{7}{|l|}{ Self-care } \\
\hline I & $629(92)$ & $\mathrm{I}, \mathrm{II} 7$ (95) & I 36 (88) & $239(93)$ & 0.117 & 0.039 \\
\hline 2 & $48(7)$ & $57(5)$ & $18(12)$ & $16(6)$ & & \\
\hline 3 & $2(0.3)$ & $3(0.3)$ & - & I (0.4) & & \\
\hline \multicolumn{7}{|l|}{ Usual activities } \\
\hline I & $545(80)$ & $\mathrm{I}, 027(87)$ & $100(65)$ & $198(77)$ & 0.024 & $<0.001$ \\
\hline 2 & 117 (I7) & $132(1 \mid)$ & $49(32)$ & $53(21)$ & & \\
\hline 3 & $17(3)$ & $18(1.5)$ & $5(3)$ & $5(2)$ & & \\
\hline \multicolumn{7}{|l|}{ Pain/discomfort } \\
\hline I & $430(63)$ & $894(76)$ & $73(47)$ & $160(63)$ & 0.001 & $<0.001$ \\
\hline 2 & $214(31)$ & $244(2 \mathrm{I})$ & $60(39)$ & $82(32)$ & & \\
\hline 3 & $35(5)$ & $39(3)$ & $21(14)$ & $13(5)$ & & \\
\hline \multicolumn{7}{|l|}{ Anxiety/depression } \\
\hline 1 & $560(82)$ & I,063 (90) & III (72) & $207(8 I)$ & 0.118 & $<0.001$ \\
\hline 2 & $108(16)$ & $106(9)$ & $38(25)$ & $43(17)$ & & \\
\hline 3 & II (2) & $8(1)$ & $5(3)$ & $6(2)$ & & \\
\hline EQ-VAS & $71.4 \pm 20.2$ & $74.1 \pm 17.5$ & $65.4 \pm 22.9$ & $68.3 \pm 18.7$ & 0.185 & $<0.001$ \\
\hline
\end{tabular}

Notes: Data are presented as mean \pm SD or n (\%). *Comparison between S- and S+ groups within the W+ groups. **Comparison among the four groups. "--" indicates no data. Abbreviations: EQ-5D, EuroQoL five dimensions questionnaire; EQ-VAS, EQ-5D visual analogue scale; S, smoking; W, wheezing.

ACO groups were prescribed more respiratory medicine compared to both $\mathrm{W}-$ groups $(P<0.001)$. Within the ACO groups, the prescription pattern was slightly different according to the smoking history. The asthma-predominant ACO group was prescribed more inhaled corticosteroids (ICS)/ long-acting beta-agonists (LABA). Conversely, the COPDpredominant $\mathrm{ACO}$ group was prescribed more long-acting muscarinic antagonists (LAMA) (Table 4).

Multiple logistic regression analysis showed that, when the OR of the W-/S- group was set to 1, the COPD-predominant ACO group was associated with increased OR of any exacerbation (age, sex, BMI, household income, and $\mathrm{FEV}_{1} \%$ predicted - adjusted OR [aOR], 1.79; 95\% CI, 1.12-2.85). However, when OR of the asthma-predominant ACO group was set to 1, the COPD-predominant ACO group did not show significant results (Table 5). When the OR of
$\mathrm{W}-/ \mathrm{S}+$ group was set to 1 , the COPD-predominant ACO group was associated with increased odds of any exacerbation (OR 2.11, 95\% CI 1.43-3.10) (Table 6).

Compared to the $\mathrm{W}-/ \mathrm{S}-$ group $(\mathrm{OR}, 1)$, outpatient and total medical costs were significantly associated with the COPD-predominant ACO group in all adjusted models (Table 7). Compared to the asthma-predominant ACO group, only outpatient medical costs were associated with the COPDpredominant ACO group in all adjusted models (Table 8).

\section{Discussion}

We identified the heterogeneity of ACO according to smoking status and found that COPD-predominant ACO might have larger clinical impact, such as lower lung function and higher outpatient medical cost than asthma-predominant ACO. Since our study subjects were selected randomly from a 
Table 3 Health care utilization and insurance-related data among the four groups

\begin{tabular}{|c|c|c|c|c|c|c|}
\hline \multirow{2}{*}{$\begin{array}{l}\text { Wheezing } \\
\text { Smoking history }\end{array}$} & \multicolumn{2}{|l|}{$\mathbf{W}-$} & \multicolumn{2}{|l|}{$\mathbf{W}+$} & \multirow[t]{2}{*}{$P$-value* } & \multirow[t]{2}{*}{$P$-value*** } \\
\hline & S- & $\mathbf{S}+$ & S- & $\mathbf{S}+$ & & \\
\hline Number of subjects, $n$ & 681 & 1,178 & 154 & 256 & & \\
\hline Insurance type & & & & & 0.873 & 0.695 \\
\hline Health insurance & $642(94)$ & $\mathrm{I}, \mathrm{I} 24(95)$ & 145 (94) & $242(95)$ & & \\
\hline Medical aid & $39(6)$ & $54(5)$ & $9(6)$ & $14(5)$ & & \\
\hline Hospitalization & & & & & 0.937 & $<0.001$ \\
\hline Never & $588(86)$ & I,070 (9I) & $128(83)$ & $212(83)$ & & \\
\hline Ever & $93(14)$ & $108(9)$ & $26(17)$ & $44(17)$ & & \\
\hline ICU admission & & & & & 0.770 & 0.970 \\
\hline Never & 671 (99) & $\mathrm{I}, \mathrm{I} 6 \mathrm{I}(99)$ & I5I (98) & $252(98)$ & & \\
\hline Ever & $10(1)$ & $17(I)$ & $3(2)$ & $4(2)$ & & \\
\hline Mean number of ER visits & $1.1 \pm 0.45$ & $1.4 \pm 0.9$ & $1.1 \pm 0.33$ & $1.4 \pm 0.67$ & 0.225 & 0.307 \\
\hline Never & $657(96)$ & $\mathrm{I}, \mathrm{I} 48$ (97) & 145 (94) & $244(95)$ & 0.607 & 0.077 \\
\hline Ever & $24(4)$ & $30(3)$ & $9(6)$ & $12(5)$ & & \\
\hline $\mathrm{I}-2$ & $22(3)$ & $22(2)$ & $8(5)$ & $8(3)$ & & \\
\hline $3-4$ & $I(0.1)$ & $5(0.4)$ & $\mathrm{I}(0.6)$ & $3(1)$ & & \\
\hline$\geq 5$ & $\mathrm{I}(0.1)$ & $2(0.2)$ & - & $\mathrm{I}(0.4)$ & & \\
\hline \multicolumn{7}{|l|}{ Mean medical costs (USD) } \\
\hline Outpatients & $19 \pm 106$ & $47 \pm 339$ & $70 \pm 182$ & $157 \pm 616$ & 0.036 & $<0.001$ \\
\hline Hospitalization & $489 \pm 1,991$ & $399 \pm 1,975$ & $55 I \pm I, 873$ & $642 \pm 2,343$ & 0.666 & 0.306 \\
\hline Total & $508 \pm 2,003$ & $445 \pm 2,080$ & $62 I \pm I, 894$ & $798 \pm 2,696$ & 0.435 & 0.102 \\
\hline \multicolumn{7}{|c|}{ Health care utilization (days) } \\
\hline Outpatient visits & $7.6 \pm 16.6$ & $7.6 \pm 13.8$ & $13.1 \pm 19.5$ & $15.9 \pm 21.9$ & 0.467 & 0.001 \\
\hline Hospitalization & $21.6 \pm 25.5$ & $19.5 \pm 19.0$ & $17.5 \pm 14.9$ & $22.9 \pm 28.4$ & 0.302 & 0.718 \\
\hline Total duration & $17.5 \pm 24.7$ & $14.7 \pm 20.2$ & $\mid 7.4 \pm 21.0$ & $23.1 \pm 32.8$ & 0.179 & 0.040 \\
\hline
\end{tabular}

Notes: Data are presented as mean \pm SD or number (\%). *Comparison between S- and S+ groups within the W+ groups. **Comparison among the four groups. “-” indicates no data.

Abbreviations: ER, emergency room; ICU, intensive care unit; S, smoking; USD, US dollars; W, wheezing.

nationwide survey, and mostly presented only mild-tomoderate airflow limitation, although their $\mathrm{FEV}_{1} / \mathrm{FVC}$ was $<0.7$. Therefore, we defined airway hyper-responsiveness as the presence of self-reported wheezing instead of the bronchial provocation test and/or bronchodilator responsiveness. Nonetheless, the clinical characteristics were well characterized when divided into the four groups
(W-/S-, $\mathrm{W}-/ \mathrm{S}+, \mathrm{W}+/ \mathrm{S}-$, and $\mathrm{W}+/ \mathrm{S}+$ ) according to smoking and wheezing status. In this study, ACO $(\mathrm{W}+)$ groups had a younger mean age, lower self-reported health and QoL levels, and higher health care utilization than $\mathrm{W}$ - groups. $\mathrm{S}+$ groups showed more frequent married status and more intensive physical activity and less common osteoarthritis, osteoporosis, and depression than S- groups, which was

Table 4 Prescription patterns of respiratory medication among the four groups

\begin{tabular}{|c|c|c|c|c|c|c|}
\hline \multirow{2}{*}{$\begin{array}{l}\text { Wheezing } \\
\text { Smoking history }\end{array}$} & \multicolumn{2}{|l|}{$\mathbf{W}-$} & \multicolumn{2}{|l|}{$\mathbf{W}+$} & \multirow[t]{2}{*}{ P-value* } & \multirow[t]{2}{*}{$P$-value** } \\
\hline & S- & $\mathbf{S}+$ & S- & $\mathbf{S}+$ & & \\
\hline Number of subjects, $n$ & 681 & $\mathrm{I}, \mathrm{I} 78$ & 154 & 256 & & \\
\hline ICS & $12(1.8)$ & $15(1.3)$ & $7(4.5)$ & II (4.3) & 0.905 & 0.002 \\
\hline ICS/LABA & II (I.6) & $33(2.8)$ & $26(16.9)$ & $27(10.5)$ & 0.064 & $<0.001$ \\
\hline LAMA & $12(1.8)$ & $38(3.2)$ & $10(6.5)$ & $21(8.2)$ & 0.526 & $<0.001$ \\
\hline LTRA & $10(1.5)$ & $25(2.1)$ & $14(9.1)$ & $21(8.2)$ & 0.755 & $<0.00 \mathrm{I}$ \\
\hline OCS & $31(4.6)$ & $43(3.7)$ & $15(9.7)$ & $29(11.3)$ & 0.615 & $<0.001$ \\
\hline SAMA & $16(2.3)$ & $27(2.3)$ & $6(3.9)$ & $15(5.9)$ & 0.383 & 0.012 \\
\hline SABA & $21(3.1)$ & $43(3.7)$ & $16(10.4)$ & $35(13.7)$ & 0.329 & $<0.001$ \\
\hline Systemic bronchodilator & $5 I(7.5)$ & $77(6.6)$ & $30(19.5)$ & $47(18.4)$ & 0.778 & $<0.001$ \\
\hline Theophylline & $54(7.9)$ & $99(8.5)$ & $35(22.7)$ & $67(26.2)$ & 0.435 & $<0.001$ \\
\hline
\end{tabular}

Notes: Data are presented as number (\%). *Comparison between S- and S+ groups within the W+ groups. **Comparison among the four groups.

Abbreviations: ICS, inhaled corticosteroids; LABA, long-acting beta-2 agonists; LAMA, long-acting muscarinic antagonists; LTRA, leukotriene receptor antagonist; OCS, oral corticosteroids; S, smoking; SABA, short-acting beta-2 agonists; SAMA, short-acting muscarinic antagonists; W, wheezing. 
Table 5 Associations of each group with exacerbations

\begin{tabular}{|c|c|c|c|c|c|c|}
\hline \multirow[t]{2}{*}{$\begin{array}{l}\text { Model } \\
\text { step }\end{array}$} & \multicolumn{3}{|c|}{$\begin{array}{l}\text { Exacerbations for four } \\
\text { groups }\end{array}$} & \multicolumn{3}{|c|}{$\begin{array}{l}\text { Exacerbations for two } \\
\text { groups }\end{array}$} \\
\hline & OR & $95 \% \mathrm{Cl}$ & $P$-value & OR & $95 \% \mathrm{Cl}$ & $P$-value \\
\hline \multicolumn{7}{|l|}{ Model I } \\
\hline W-IS- & I & & & & & \\
\hline W-/S+ & 0.65 & $0.48-0.87$ & 0.004 & & & \\
\hline $\mathrm{W}+/ \mathrm{S}_{-}$ & 1.28 & $0.80-2.07$ & 0.302 & I & & \\
\hline $\mathrm{W}+/ \mathrm{S}+$ & 1.31 & $0.89-1.94$ & 0.174 & 1.02 & $0.6-1.74$ & 0.937 \\
\hline \multicolumn{7}{|l|}{ Model 2} \\
\hline W-/S- & 1 & & & & & \\
\hline W-/S+ & 0.87 & $0.59-1.29$ & 0.494 & & & \\
\hline$W+/ S-$ & 1.14 & $0.7-1.85$ & 0.606 & I & & \\
\hline$W+/ S+$ & 1.83 & $1.16-2.88$ & 0.009 & 2.13 & $0.94-4.84$ & 0.071 \\
\hline \multicolumn{7}{|l|}{ Model 3} \\
\hline W-/S- & 1 & & & & & \\
\hline W-/S+ & 0.86 & $0.58-1.28$ & 0.463 & & & \\
\hline$W+/ S-$ & 1.13 & $0.69-1.84$ & 0.639 & I & & \\
\hline$W+/ S+$ & 1.93 & $1.22-3.05$ & 0.005 & 2.21 & $0.97-5.04$ & 0.061 \\
\hline \multicolumn{7}{|l|}{ Model 4} \\
\hline W-/S- & 1 & & & & & \\
\hline W-/S+ & 0.87 & $0.59-1.29$ & 0.489 & & & \\
\hline $\mathrm{W}+/ \mathrm{S}_{-}$ & 1.03 & $0.63-1.7$ & 0.903 & I & & \\
\hline $\mathrm{W}+/ \mathrm{S}+$ & 1.79 & I.12-2.85 & 0.015 & 2.24 & $0.97-5.16$ & 0.058 \\
\hline
\end{tabular}

Notes: Model I: unadjusted. Model 2: adjusted for age, sex, and BMI. Model 3: adjusted for age, sex, BMI, and household income. Model 4: adjusted for age, sex, $\mathrm{BMI}$, household income, and forced expiratory volume in I second.

Abbreviations: $\mathrm{BMI}$, body mass index; $\mathrm{Cl}$, confidence interval; OR, odds ratio; $\mathrm{S}$, smoking; W, wheezing.

mainly due to differences in sex between the two groups. Almost all $(1,363 / 1,434,95 \%)$ of the $\mathrm{S}+$ groups were males, and the majority of the S- groups were females (568/835, $68 \%$ ). When comparing both ACO groups, males predominated, physical activity was more intensive, and $\mathrm{FEV}_{1} / \mathrm{FVC}$ was lower in the COPD-predominant $\mathrm{ACO}$ group than in the asthma-predominant ACO group. Socioeconomic status parameters, such as household income and education levels, self-rated health, and health-related
QoL levels, were lower in the asthma-predominant ACO group than in the COPD-predominant ACO group. The asthma-predominant ACO group showed higher prevalence of osteoporosis compared to the COPD-predominant ACO group. This may be due to the higher proportion of women in the asthma-predominant ACO group. There was no difference between the asthma-predominant ACO and the COPD-predominant ACO in the medical utilization, except outpatient costs, which were higher in the COPD-predominant ACO group. Multiple logistic regression analysis showed that the COPD-predominant ACO group was associated with increased odds of exacerbations compared to both groups without wheezing (W-/S- and W-/ $\mathrm{S}+)$ but not the asthma-predominant ACO group.

Subjects with ACO $(\mathrm{W}+)$ were more likely to experience exacerbations and to have lower health-related QoL compared to those without ACO (W-). Both $\mathrm{W}+$ groups also showed higher medical costs and health care utilization than $\mathrm{W}-$ groups. Similarly, most previous comparative studies of ACO and COPD showed that ACO had more frequent exacerbations, symptoms, and comorbidities and lower QoL. ${ }^{6,24-28}$ In the EPI-SCAN study of a Spanish cohort, more frequent respiratory symptoms and exacerbations and lower $\mathrm{FEV}_{1}, \mathrm{FVC}$, QoL, and physical activities were observed in ACO patients than in COPD patients. ${ }^{10}$ The PLATINO study of Latin American countries also showed that ACO patients had more symptoms, lower pulmonary function, higher risk of exacerbations and hospitalizations, and worse health status than those with COPD. ${ }^{29}$ These results were similarly observed in the COPDGene Study conducted in the USA and South Korea. ${ }^{9,13}$ In a meta-analysis of ACO-related studies, no differences in sex, smoking amounts, lung function, and 6-minute walk distance were found between ACO and COPD patients, but

Table 6 Associations of the four subgroups or each variable with any exacerbation requiring hospitalization or emergency room visit

\begin{tabular}{|c|c|c|c|c|c|c|c|c|c|c|c|c|}
\hline Variables & OR & $95 \% \mathrm{Cl}$ & $P$-value & OR & $95 \% \mathrm{Cl}$ & $P$-value & OR & $95 \% \mathrm{Cl}$ & $P$-value & OR & $95 \% \mathrm{Cl}$ & $P$-value \\
\hline W-/S- & 1 & & & 1.33 & $0.85-2.1$ & 0.217 & 0.88 & $0.54-1.44$ & 0.617 & 0.63 & $0.38-1.05$ & 0.077 \\
\hline $\mathrm{W}-/ \mathrm{S}+$ & 0.75 & $0.48-1.18$ & 0.217 & I & & & 0.66 & $0.36-1.22$ & 0.185 & 0.48 & $0.32-0.7$ & $<0.001$ \\
\hline $\mathrm{W}+/ \mathrm{S}-$ & 1.13 & $0.70-1.84$ & 0.617 & 1.51 & $0.82-2.77$ & 0.185 & 1 & & & 0.72 & $0.38-1.37$ & 0.311 \\
\hline $\mathrm{W}+/ \mathrm{S}+$ & 1.58 & $0.95-2.63$ & 0.077 & 2.11 & $1.43-3.10$ & $<0.001$ & 1.40 & $0.73-2.66$ & 0.311 & 1 & & \\
\hline Male & 0.58 & $0.4-0.85$ & 0.005 & & & & & & & & & \\
\hline Age (years) & 1.01 & $1.00-1.02$ & 0.194 & & & & & & & & & \\
\hline Smoking (pack-years) & 1.01 & $1.00-1.01$ & 0.187 & & & & & & & & & \\
\hline \multicolumn{13}{|l|}{ BMI } \\
\hline Underweight & 2.62 & $1.47-4.67$ & 0.001 & & & & & & & & & \\
\hline Normal & I & & & & & & & & & & & \\
\hline Overweight & 0.80 & $0.59-1.09$ & 0.155 & & & & & & & & & \\
\hline Obesity & 0.74 & $0.26-2.15$ & 0.584 & & & & & & & & & \\
\hline
\end{tabular}

Notes: Underweight $=\mathrm{BMI}<18.5 \mathrm{~kg} / \mathrm{m}^{2}$. Normal $=18.5 \leq \mathrm{BMI}<25 \mathrm{~kg} / \mathrm{m}^{2}$. Overweight $=25 \leq \mathrm{BMl}<30 \mathrm{~kg} / \mathrm{m}^{2}$. Obesity $=\mathrm{BMl} \geq 30 \mathrm{~kg} / \mathrm{m}^{2}$.

Abbreviations: $\mathrm{BMI}$, body mass index; $\mathrm{Cl}$, confidence interval; OR, odds ratio; $\mathrm{S}$, smoking; $\mathrm{W}$, wheezing. 
Table 7 Associations of the four groups with medical cost

\begin{tabular}{|c|c|c|c|c|c|c|}
\hline \multirow[t]{2}{*}{$\begin{array}{l}\text { Model } \\
\text { step }\end{array}$} & \multicolumn{2}{|c|}{ Outpatient } & \multicolumn{2}{|c|}{ Inpatient } & \multicolumn{2}{|c|}{$\begin{array}{l}\text { Total medical } \\
\text { cost }\end{array}$} \\
\hline & Beta & $P$-value & Beta & $P$-value & Beta & $P$-value \\
\hline \multicolumn{7}{|l|}{ Model I } \\
\hline \multicolumn{7}{|l|}{ W-/S- } \\
\hline W-/S+ & 27,634 & $0.08 I$ & $-90,308$ & 0.353 & $-62,674$ & 0.540 \\
\hline$W_{+} / S_{-}$ & 50,708 & 0.084 & 62,183 & 0.730 & II 2,890 & 0.552 \\
\hline$W+/ S+$ & 137,557 & $<0.001$ & 152,935 & 0.301 & 290,492 & 0.062 \\
\hline \multicolumn{7}{|l|}{ Model 2} \\
\hline \multicolumn{7}{|l|}{ W-IS- } \\
\hline W-/S+ & 29,739 & 0.145 & 52,868 & 0.673 & 82,606 & 0.530 \\
\hline$W+/ S-$ & 51,133 & 0.083 & 25,689 & 0.887 & 76,822 & 0.687 \\
\hline$W+/ S+$ & 145,122 & $<0.001$ & 307,186 & 0.061 & 452,309 & 0.009 \\
\hline \multicolumn{7}{|l|}{ Model 3} \\
\hline \multicolumn{7}{|l|}{ W-IS- } \\
\hline W-/S+ & 27,691 & 0.184 & 67,490 & 0.596 & 95,181 & 0.477 \\
\hline$W+/ S-$ & 47,630 & 0.117 & 18,783 & 0.919 & 66,414 & 0.734 \\
\hline$W+/ S+$ & $\mid 46,848$ & $<0.001$ & 346,877 & 0.038 & 493,726 & 0.005 \\
\hline \multicolumn{7}{|l|}{ Model 4} \\
\hline \multicolumn{7}{|l|}{ W-/S- } \\
\hline W-/S+ & 33,058 & 0.111 & 103,726 & 0.413 & 136,786 & 0.304 \\
\hline$W+/ S-$ & 37,503 & 0.216 & $-49,585$ & 0.789 & $-12,083$ & 0.950 \\
\hline$W+/ S+$ & I 38,474 & $<0.001$ & 290,345 & 0.081 & 428,819 & 0.014 \\
\hline
\end{tabular}

Notes: Model I: unadjusted. Model 2: adjusted for age, sex, and BMI. Model 3: adjusted for age, sex, BMI, and household income. Model 4: adjusted for age, sex, $\mathrm{BMI}$, household income, and forced expiratory volume in I second.

Abbreviations: BMI, body mass index; S, smoking; W, wheezing.

ACO patients were younger and had higher BMI, greater use of medical resources, and lower health-related QoL. ${ }^{30}$ In terms of medical use and cost, Medicare beneficiary data revealed that patients with diagnostic codes of both COPD and asthma had higher medical use and expenses than those with codes

Table 8 Association of wheezing subtypes with medical cost

\begin{tabular}{|c|c|c|c|c|c|c|}
\hline \multirow[t]{2}{*}{ Variables } & \multicolumn{2}{|c|}{ Outpatient } & \multicolumn{2}{|c|}{ Inpatient } & \multicolumn{2}{|c|}{$\begin{array}{l}\text { Total medical } \\
\text { cost }\end{array}$} \\
\hline & Beta & $P$-value & Beta & $P$-value & Beta & $P$-value \\
\hline \multicolumn{7}{|l|}{ Model I } \\
\hline \multicolumn{7}{|l|}{ W-/S+ } \\
\hline$W+/ S+$ & 86,849 & 0.089 & 90,752 & 0.683 & $|77,60|$ & 0.473 \\
\hline \multicolumn{7}{|l|}{ Model 2} \\
\hline \multicolumn{7}{|l|}{ W-/S+ } \\
\hline $\mathrm{W}+/ \mathrm{S}+$ & 162,235 & 0.027 & 295,827 & 0.356 & 458,062 & 0.198 \\
\hline \multicolumn{7}{|l|}{ Model 3} \\
\hline \multicolumn{7}{|l|}{ W-/S+ } \\
\hline W+/S+ & 192,723 & 0.011 & 287,907 & 0.385 & 480,630 & 0.191 \\
\hline \multicolumn{7}{|l|}{ Model 4} \\
\hline \multicolumn{7}{|l|}{ W-IS+ } \\
\hline$W+/ S+$ & 203,598 & 0.006 & 304,47 I & 0.358 & 508,068 & 0.166 \\
\hline
\end{tabular}

Notes: Model I: unadjusted. Model 2: adjusted for age, sex, and BMI. Model 3: adjusted for age, sex, BMI, and household income. Model 4: adjusted for age, sex, $\mathrm{BMI}$, household income, and forced expiratory volume in I second.

Abbreviations: BMI, body mass index; S, smoking; W, wheezing. of COPD alone. ${ }^{14}$ In Korea, medical utilization and cost were reported to be significantly higher in ACO than in COPD or asthma. ${ }^{13,31}$ Systematic reviews and meta-analyses have also revealed more medical use including emergency department visits and hospitalization for ACO patients. . $^{30,32}$

In contrast to these previous studies, Cosio et a ${ }^{12}$ reported that ACO patients had better survival than COPD patients. In addition, a recent study reported that $\mathrm{ACO}$ with early onset of asthma has a better prognosis than COPD. However, ACO with late onset of asthma had poorer prognosis compared to COPD. ${ }^{11}$ The collective data indicate that there may be heterogeneous groups within ACO patients and the prognosis can be determined accordingly. The aforementioned study showed that the outcome of ACO patients varies according to the time of asthma diagnosis, and our study demonstrated the heterogeneity of ACO according to smoking history. Although the COPD-predominant ACO group had higher household income, education levels, and health-related QoL than the asthma-predominant ACO group, COPD-predominant ACO was associated with higher outpatient medical cost compared to the asthma-predominant ACO group. Multivariate logistic regression analysis adjusted for age, sex, BMI, household income, and $\mathrm{FEV}_{1}$ showed that the COPD-predominant $\mathrm{ACO}$ was associated with a higher rate of hospitalization than the asthma-predominant ACO group, although not statistically significant.

There are some limitations to this study. First, we used arbitrary definitions for the four groups, which did not fully meet the common diagnostic criteria. Because we used KNHANES and NHI data, bronchial provocation test or bronchodilator responsiveness and other respiratory imaging studies were not included. Therefore, the distinction between asthma and ACO proposed in this study were not accurate. It is also difficult to reflect the asthma control status, severity, or medication compliance. For example, asthma symptoms or control can vary depending on how steady the asthma medication is used and it may misclassified to a group of wheezing $(-)$ or $(+)$. This inaccurate assignment is likely to have weakened the statistical power of this study. Second, pulmonary function was measured only once at a specific time point and it could be affected by condition or short-term disease such as upper respiratory infection of patients. This may also be an obstacle to the precise distinction between $\mathrm{W}+$ and $\mathrm{W}-$. However, many previous population-based studies have used "wheezing" as a representative symptom of asthma. ${ }^{22,31,33,34}$ To overcome these limitations, we used NHI data in conjunction with KNHANES data. NHI data reflect the actual illness and prescription, which can complement the aforementioned drawbacks. As confirmed in the study 
results, this classification seemed to represent two diseases to some extent, because the prescription patterns according to this classification were quite characteristic. In addition, it was a nationwide survey that had the advantage of being able to represent the whole population of a country and that included important variables associated with disease, such as socioeconomic status and QoL. Also, this study benefited from obtaining relatively accurate data on medical use and expenses by using NHI claim data. Second, although statistical analysis was performed by adjusting for sex through multiple logistic regression, sex difference associated with smoking history might be too large to overcome. Therefore, some of our results may be due to sex differences between groups and not differences between ACO subtypes.

Particularly, within ACO groups, two subtypes with distinct characteristics were found and they also showed marked differences in terms of exacerbation frequency and medical costs. While asthma-predominant ACO individuals displayed poorer socioeconomic status and QoL compared to the COPD-predominant ACO group, the COPD-predominant ACO group showed higher medical use and expenditure than the asthma-predominant ACO group. In this study, we found that ACO has more exacerbations than COPD with mild airway obstruction and that it can show various phenotypes with different prognoses. Therefore, in order to manage ACO patients well, it is necessary to recognize the characteristics well and appropriately allocate limited medical resources.

\section{Acknowledgments}

This research was supported by the intramural research promotion grants from Ewha Womans University School of Medicine, Korea Centers for Disease Control and Prevention (2014-E33003-00), and National Research Foundation of Korea Grant funded by the Korean Government (20100027945). HIRA provided the NHI claim database and merged them with data from the Korean National Health and Nutritional Examination Survey (KNHANES).

\section{Disclosure}

CK Rhee received consulting/lecture fees from MSD, AstraZeneca, Novartis, GSK, Takeda, Mundipharma, Sandoz, Boehringer-Ingelheim, and Teva-Handok. The other authors report no conflicts of interest in this work.

\section{References}

1. Rhee CK. Phenotype of asthma-chronic obstructive pulmonary disease overlap syndrome. Korean J Intern Med. 2015;30:443-449.

2. Kim SR, Rhee YK. Overlap between asthma and COPD: where the two diseases converge. Allergy Asthma Immunol Res. 2010;2:209-214.
3. Papaiwannou A, Zarogoulidis P, Porpodis K, et al. Asthma-chronic obstructive pulmonary disease overlap syndrome (ACOS): current literature review. J Thorac Dis. 2014;6(suppl 1):S146-S151.

4. Postma DS, Rabe KF. The asthma-COPD overlap syndrome. NEnglJMed. 2015;373:1241-1249.

5. Cazzola M, Rogliani P. Do we really need asthma-chronic obstructive pulmonary disease overlap syndrome? J Allergy Clin Immunol. 2016; 138:977-983.

6. Sin DD. Asthma-COPD overlap syndrome: what we know and what we don't. Tuberc Respir Dis. 2017;80:11-20.

7. Caillaud D, Chanez P, Escamilla R, et al. Asthma-COPD overlap syndrome (ACOS) vs 'pure' COPD: a distinct phenotype? Allergy. 2017;72: $137-145$.

8. Ding B, DiBonaventura M, Karlsson N, Ling X. Asthma-chronic obstructive pulmonary disease overlap syndrome in the urban Chinese population: prevalence and disease burden using the 2010, 2012, and 2013 China national health and wellness surveys. Int J Chron Obstruct Pulmon Dis. 2016;11:1139-1150.

9. Hardin M, Silverman EK, Barr RG, et al. The clinical features of the overlap between COPD and asthma. Respir Res. 2011;12:127.

10. Miravitlles M, Soriano JB, Ancochea J, et al. Characterisation of the overlap COPD-asthma phenotype. Focus on physical activity and health status. Respir Med. 2013;107:1053-1060.

11. Lange P, Colak Y, Ingebrigtsen TS, Vestbo J, Marott JL. Long-term prognosis of asthma, chronic obstructive pulmonary disease, and asthma-chronic obstructive pulmonary disease overlap in the Copenhagen city heart study: a prospective population-based analysis. Lancet Respir Med. 2016;4:454-462.

12. Cosio BG, Soriano JB, Lopez-Campos JL, et al. Defining the asthmaCOPD overlap syndrome in a COPD cohort. Chest. 2016;149:45-52.

13. Rhee $\mathrm{CK}$, Yoon $\mathrm{HK}$, Yoo $\mathrm{KH}$, et al. Medical utilization and cost in patients with overlap syndrome of chronic obstructive pulmonary disease and asthma. COPD. 2014;11:163-170.

14. Blanchette CM, Gutierrez B, Ory C, Chang E, Akazawa M. Economic burden in direct costs of concomitant chronic obstructive pulmonary disease and asthma in a Medicare advantage population. J Manag Care Pharm. 2008;14:176-185.

15. Kweon S, Kim Y, Jang MJ, et al. Data resource profile: the Korea National Health and Nutrition Examination Survey (KNHANES). Int J Epidemiol. 2014;43:69-77.

16. Shopland DR, Hartman AM, Gibson JT, Mueller MD, Kessler LG, Lynn WR. Cigarette smoking among U.S. Adults by state and region: estimates from the current population survey. J Natl Cancer Inst. 1996;88: 1748-1758.

17. Wingo PA, Ries LAG, Giovino GA, et al. Annual report to the nation on the status of cancer, 1973-1996, with a special section on lung cancer and tobacco smoking. J Natl Cancer Inst. 1999;91:675-690.

18. Lee H, Rhee CK, Lee BJ, et al. Impacts of coexisting bronchial asthma on severe exacerbations in mild-to-moderate COPD: results from a national database. Int J Chron Obstruct Pulmon Dis. 2016;11:775-783.

19. Kim J, Rhee CK, Yoo KH, et al. The health care burden of high grade chronic obstructive pulmonary disease in Korea: analysis of the Korean health insurance review and assessment service data. Int J Chron Obstruct Pulmon Dis. 2013;8:561-568.

20. Kim J, Lee JH, Kim Y, et al. Association between chronic obstructive pulmonary disease and gastroesophageal reflux disease: a national cross-sectional cohort study. BMC Pulm Med. 2013;13:51.

21. Kim C, Yoo KH, Rhee CK, et al. Health care use and economic burden of patients with diagnosed chronic obstructive pulmonary disease in Korea. Int J Tuberc Lung Dis. 2014;18:737-743.

22. Lee JH, Rhee CK, Kim K, et al. Identification of subtypes in subjects with mild-to-moderate airflow limitation and its clinical and socioeconomic implications. Int J Chron Obstruct Pulmon Dis. 2017;12:1135-1144.

23. EuroQol Group. Euroqol - a new facility for the measurement of healthrelated quality of life. Health Policy. 1990;16:199-208.

24. Barnes PJ. Asthma-COPD overlap. Chest. 2016;149:7-8. 
25. Gibson PG, McDonald VM. Asthma-COPD overlap 2015: now we are six. Thorax. 2015;70:683-691.

26. Hardin M, Cho M, McDonald ML, et al. The clinical and genetic features of COPD-asthma overlap syndrome. Eur Respir J. 2014;44:341-350.

27. Barrecheguren M, Esquinas C, Miravitlles M. The asthma-chronic obstructive pulmonary disease overlap syndrome (ACOS): opportunities and challenges. Curr Opin Pulm Med. 2015;21:74-79.

28. Chung JW, Kong KA, Lee JH, Lee SJ, Ryu YJ, Chang JH. Characteristics and self-rated health of overlap syndrome. Int J Chron Obstruct Pulmon Dis. 2014;9:795-804.

29. Menezes AM, Montes de Oca M, Perez-Padilla R, et al. Increased risk of exacerbation and hospitalization in subjects with an overlap phenotype: COPD-asthma. Chest. 2014;145:297-304.

30. Alshabanat A, Zafari Z, Albanyan O, Dairi M, FitzGerald JM. Asthma and COPD overlap syndrome (ACOS): a systematic review and meta analysis. PLoS One. 2015;10:e0136065.
31. Kim J, Kim YS, Kim K, et al. Socioeconomic impact of asthma, chronic obstructive pulmonary disease and asthma-COPD overlap syndrome. J Thorac Dis. 2017;9:1547-1556.

32. Nielsen M, Barnes CB, Ulrik CS. Clinical characteristics of the asthmaCOPD overlap syndrome - a systematic review. Int J Chron Obstruct Pulmon Dis. 2015;10:1443-1454.

33. Arif AA, Delclos GL, Lee ES, Tortolero SR, Whitehead LW. Prevalence and risk factors of asthma and wheezing among us adults: an analysis of the NHANES III data. Eur Respir J. 2003;21:827-833.

34. Xuan W, Peat JK, Toelle BG, Marks GB, Berry G, Woolcock AJ. Lung function growth and its relation to airway hyperresponsiveness and recent wheeze. Results from a longitudinal population study. Am J Respir Crit Care Med. 2000;161:1820-1824.

\section{Publish your work in this journal}

The International Journal of COPD is an international, peer-reviewed journal of therapeutics and pharmacology focusing on concise rapid reporting of clinical studies and reviews in COPD. Special focus is given to the pathophysiological processes underlying the disease, intervention programs, patient focused education, and self management protocols.

\section{Dovepress}

This journal is indexed on PubMed Central, MedLine and CAS. The manuscript management system is completely online and includes a very quick and fair peer-review system, which is all easy to use. Visit $\mathrm{http} / / / \mathrm{www}$.dovepress.com/testimonials.php to read real quotes from published authors. 